\title{
CARACTERÍSTICAS OCEANOGRÁFICAS Y BIOLÓGICAS DE LAS COMUNIDADES DEL PLANCTON Y NECTON DEL ÁREA MARINA COSTERA PROTEGIDA FRANCISCO COLOANE: UNA REVISIÓN
}

\author{
OCEANOGRAPHIC AND BIOLOGICAL CHARACTERISTICS OF THE PLANKTON AND NECTON \\ COMMUNITIES IN THE COASTAL MARINE PROTECTED AREA FRANCISCO COLOANE: A \\ REVIEW \\ Daniela Haro ${ }^{1,2}$, Anelio Aguayo-Lobo ${ }^{3} \&$ Jorge Acevedo $^{4}$
}

\begin{abstract}
The objective of this study was to make a review of the current state of knowledge on the Coastal Marine Protected Area (CMPA) Francisco Coloane in terms of the physical and chemical oceanography, the phyto-zooplankton compositions and the fish, birds and marine mammals species present, and provide temperature and salinity data collected during a marine survey carried out the 2012 fall season. Surface temperatures fluctuate between 2.0 and $9.0^{\circ} \mathrm{C}$. Salinity varies between 23 and 30.9 PSU. The most oxygenated waters and those of highest $\mathrm{pH}$ were recorded within Seno Ballena (320-340 $\mathrm{mmoles} \mathrm{Kg}^{-1}$; 8.11 units of $\mathrm{pH}$ ). In spring nutrient levels indicate low surface concentrations, except for phosphate, the phytoplankton is dominated by diatoms and the zooplankton is composed of copepods and meroplanktonic larvae with high abundance levels in the Jeronimo Channel. The family of fish most representative is the Nototheniidae, and among marine birds and mammals, the Magellan penguin and the humpback whale are the most sighted. The review indicates that it is necessary to make further studies on physical oceanography and the benthic community of CMPA.
\end{abstract}

Key words: CMPA, pelagic ecosystem, birds and marine mammals, Magallanes.

\section{RESUMEN}

El objetivo de este trabajo fue hacer una revisión sobre el conocimiento de las características oceanográficas físicas y químicas, composición del fito y zooplancton, peces, aves y mamíferos marinos

\footnotetext{
${ }^{1}$ Programa de Magíster en Ciencias mención Manejo y Conservación de Recursos Naturales en Ambientes Subantárticos. Facultad de Ciencias. Universidad de Magallanes, Punta Arenas, Chile.

${ }^{2}$ Laboratorio de Ecología y Ciencias Ambientales, Instituto de la Patagonia, Universidad de Magallanes, Punta Arenas, Chile.

${ }^{3}$ Instituto Antártico Chileno (INACH), Punta Arenas, Chile.

${ }^{4}$ Centro Regional de Estudios del Cuaternario (CEQUA), Punta Arenas, Chile.
} 
del Área Marina Costera Protegida (AMCP) Francisco Coloane, e informar sobre datos de temperatura y salinidad obtenidos en el área durante una prospección en otoño de 2012. La temperatura superficial fluctuó entre 2,0 y $9,0^{\circ} \mathrm{C}$. La salinidad varió entre 23 y 30,9 PSU. Las aguas más oxigenadas y con alto pH se registraron en seno Ballena (320-340 $\mu$ moles $\mathrm{Kg}^{-1}$ y 8,11 unidades de $\mathrm{pH}$ ). En primavera los niveles de nutrientes indican que la concentración en superficie no es alta, con excepción del fosfato, el fitoplancton está representado por diatomeas y el zooplancton por copépodos y larvas meroplanctónicas, destacando canal Jerónimo por su alta abundancia. La familia de peces más representativa fue Nototheniidae, y entre las aves y mamíferos marinos, destaca el pingüino de Magallanes y la ballena jorobada. La revisión realizada indica que es necesario hacer mayores estudios sobre la oceanografía física y la comunidad bentónica del AMCP.

Palabras clave: AMCP, ecosistema pelágico, aves y mamíferos marinos, Magallanes.

\section{INTRODUCCIÓN}

Según la Unión Internacional para la Conservación de la Naturaleza (UICN), las Áreas Marinas Costeras Protegidas (AMCP) son el espacio que incluye agua y fondo marino, rocas, playas $y$ terrenos de playa fiscales, así como flora y fauna, recursos históricos y culturales que la ley $\mathrm{u}$ otros medios colocan en reserva para proteger todo o parte del medio así delimitado (De Andrade, 2006). Estas áreas son esenciales para la conservación de la biodiversidad (Dudley, 2008) y surgen para proteger, mantener y restaurar los recursos naturales y culturales de las aguas costeras y marinas (de Andrade, 2006).

En la región de Magallanes, el AMCP Francisco Coloane constituye la primera Área Marina creada en Chile, en agosto de 2003. Esta zona cuenta con una superficie aproximada de 67.197 ha, conteniendo en su interior el Parque Marino del mismo nombre que representa una zona núcleo de 1.503 ha. El AMCP Francisco Coloane se encuentra geográficamente ubicada en la sección central del estrecho de Magallanes, a $80 \mathrm{mn}$ de la ciudad de Punta Arenas (Cabezas, 2006; Cornejo \& Kusch, 2006). El objetivo del área Francisco Coloane fue contribuir a la conservación del área de alimentación de la ballena jorobada (Megaptera novaeangliae, Borowski, 1781), la conservación de las áreas de reproducción del pingüino de Magallanes (Spheniscus magellanicus, Foster, 1781) y del lobo marino común (Otaria flavescens, Shaw 1800), la protección de las unidades ecológicas asociadas y la mantención de los servicios ecosistémicos (Ministerios de Defensa y Economía D.S. N²76; Aguayo-Lobo et al., 2011).

Una situación poco frecuente para los canales Magallánicos se presenta en el ecosistema pelágico de esta área, ya que presenta una alta diversidad de fauna, registrándose al menos 13 especies de mamíferos marinos y 32 de aves marinas y/o costeras (AguayoLobo et al. 2011; CEQUA ${ }^{1}$ ). Por otra parte, el AMCP constituye una zona de alimentación de grandes mamíferos, destacando la ballena jorobada, el lobo marino común y el lobo fino austral (Arctocephalus australis, Zimmermann, 1783). El AMCP también destaca como zona de reproducción de dos colonias de lobo marino común y de una importante colonia de pingüino de Magallanes (Cornejo \& Kusch, 2006). A pesar de la riqueza específica y de la presencia de especies emblemáticas, como la ballena jorobada, lobos marinos y pingüinos, aún no se tiene mayor conocimiento de los procesos que determinan la alta diversidad, la productividad del ecosistema y de las especies que sostienen las tramas tróficas del AMCP. La información que se tiene hasta la fecha corresponde a escasos estudios puntuales, los cuales se encuentran dispersos en la literatura publicada.

En este contexto, el objetivo del presente trabajo fue realizar una revisión sobre el conocimiento existente del ecosistema pelágico en el Área Marina Costera Protegida Francisco Coloane, con el fin de unificar en un solo trabajo la información que se dispone sobre este ecosistema en particular, de tal manera de identificar debilidades y necesidades futuras. 


\section{MATERIALES Y MÉTODOS}

Área de estudio

El AMCP Francisco Coloane se ubica en la parte central del estrecho de Magallanes, extendiéndose por el este desde bahía Cordes


$72^{\circ} 34^{\prime} \mathrm{W}$ ) por el oeste, y por el norte desde estero Núñez (5317'S; 72²6’W) hasta seno Helado

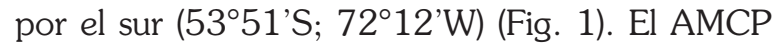
incluye paso Tortuoso, canal Jerónimo, paso Inglés, desembocadura del río Batchelor, bahías Fortescue y Cordes, mitad de seno Ballena, boca del canal Bárbara (paso Shag) y seno Helado. En esta área se ubican los glaciares de seno Ballena y seno Helado, y las islas Carlos III, Rupert, Charles, James, Mounmouth, Wet y Alcayaga, así como islotes menores.

Base de datos

La información de este trabajo proviene tanto de datos publicados del área, entre los años 1989 y 2011, como de resultados obtenidos en una prospección realizada entre el 21 y 24 de mayo de 2012, durante la ejecución del proyecto "Diagnóstico de la distribución espacio temporal de la ballena jorobada en el Área Marina Costera Protegida Francisco Coloane" de la Fundación BIOMAR. En esta campaña se realizaron tres estaciones de muestreo, canal Jerónimo (53²1'20,00”; 72²9'20,04”), isla Carlos III $\left(53^{\circ} 38^{\prime} 85,15^{\prime \prime} ; 72^{\circ} 14^{\prime} 89.80^{\prime \prime}\right)$ y paso Shag (5349'28,45"; 72¹1'18,70”), en las cuales se registraron datos de temperatura y salinidad, desde la superficie hasta una profundidad de $45 \mathrm{mts}$, con un CTD marca Seabird modelo SBE 19 seacat. Los datos fueron extraídos y procesados de acuerdo a la secuencia de procesamiento recomendada por el Centro Nacional de Datos Hidrográficos y Oceanográficos de Chile (CENDHOC).

De la información previamente publicada, se consideraron estudios sobre oceanografía física y química, composición fito y zooplanctónica, peces, aves y mamíferos marinos en el AMCP. La información analizada corresponde a 16 expediciones científicas (Tabla 1).

\section{RESULTADOS Y DISCUSIÓN}

El AMCP Francisco Coloane se ubica en la sección este de la subcuenca occidental y en la parte oeste de la subcuenca central del estrecho de Magallanes, descritas por Antezana et al. (1992). Según Pickard (1973), Panella et al. (1991), Antezana et al., (1992) y Valdenegro \& Silva, (2003), la subcuenca occidental, abarca desde la entrada del océano Pacífico hasta la isla Carlos III, lugar donde existe un umbral de $100 \mathrm{~m}$ de profundidad. Esta subcuenca tiene profundidades de hasta $1000 \mathrm{~m} \mathrm{y}$ está compuesta por aguas Subantárticas (ASAA) que ingresan desde el Pacífico, mezclándose con agua dulce del interior del continente, formando agua estuarina salada (AE-salada) y agua Subantártica modificada (ASAAM) en los 150 m superiores. Valdenegro \& Silva (2003) señalan para esta subcuenca, que la temperatura y salinidad durante primavera, alcanza $6,5{ }^{\circ} \mathrm{C}$ y $28,5 \mathrm{PSU}$ en superficie, aumentando a $9,5^{\circ} \mathrm{C}$ y 33 PSU en los $100 \mathrm{~m}$. El oxígeno disuelto y $\mathrm{pH}$, alcanza $6,5 \mathrm{ml} \cdot \mathrm{L}^{-1}\left(290 \mu\right.$ moles $\left.\mathrm{Kg}^{-1}\right)$ y 7,8 unidades, disminuyendo en profundidad.

La subcuenca central, abarca desde isla Carlos III hasta la segunda Angostura, alcanza profundidades de 500 m y está compuesta por AE-salada, las cuales se desplazan desde la cuenca occidental, sobrepasando el umbral de Carlos III, y hundiéndose debido a su densidad. Además, esta subcuenca estaría compuesta en menor grado por aguas Atlánticas, las cuales alcanzan con su influencia la zona comprendida entre Carlos III y cabo Froward. Valdenegro \& Silva, (2003) indicaron que en primavera, la temperatura en esta cuenca es relativamente homogénea $\left(7-7,5^{\circ} \mathrm{C}\right)$ y la salinidad superficial sería de 30,5 PSU aumentando a 31,5 PSU en los $500 \mathrm{~m}$. El oxígeno y pH presentan valores de 6,5 a $7 \mathrm{ml} \cdot \mathrm{L}^{-1}\left(290-313 \mu\right.$ moles $\left.\mathrm{Kg}^{-1}\right)$ y de 7,7 a 7,9 unidades de $\mathrm{pH}$, disminuyendo con la profundidad.

Por tanto las aguas del AMCP están constituidas por AE-salada, ASSAM y en menor grado por aguas con influencia Atlántica. Además, estas aguas presentarían la estructura de doble capa que caracteriza a los canales del sur de Chile, la cual es provocada por la mezcla de aguas que se produce con el ingreso de ASSA del Pacífico (Valdenegro \& Silva, 2003; Sievers, 2006). El agua superficial $(<50-$ 
Tabla 1: Lista de expediciones científicas realizadas en el estrecho de Magallanes y consideradas en esta revisión, en las cuales se realizó al menos una estación de muestreo en el AMCP Francisco Coloane.

\begin{tabular}{|c|c|c|c|c|c|}
\hline & Campaña & Año & $\begin{array}{l}\text { Número } \\
\text { Estaciones }\end{array}$ & Lugar AMCP & Fuente \\
\hline a & Expedición OGS Explora & 1989 & 1 & Carlos III & Panella et al. 1991 \\
\hline $\mathrm{b}$ & Expedición OGS Explora & 1989 & 1 & Carlos III & Uribe 1991 \\
\hline c & Expedición OGS Explora & 1989 & 1 & Carlos III & Fonda Umani \& Monti 1991 \\
\hline d & Universidad de Magallanes & 1991 & 1 & Canal Jerónimo & Andrade 1991 \\
\hline$e$ & Expedición Cariboo & 1991 & 1 & Carlos III & Carrada et al. 1994 \\
\hline f & Expedición Cariboo & 1991 & 1 & Carlos III & Zingone et al. 2011 \\
\hline$g$ & Expedición Victor Hensen & 1994 & 2 & Carlos III, islas Charles & Arntz \& Gorny 1996 \\
\hline h & Expedición Italica & 1995 & 2 & Carlos III, islas Charles & Saggiomo et al. 2011 \\
\hline i & Expedición Italica & 1995 & 1 & Islas Charles & Hamamé \& Antezana 2010 \\
\hline j & Expedición CIMAR Fiordos 3 & $1997-1998$ & 2 & Canal Jerónimo, islas Charles & Valdenegro \& Silva 2003 \\
\hline $\mathrm{k}$ & Expedición CIMAR Fiordos 3 & $1997-1998$ & 2 & Canal Jerónimo, islas Charles & Avaria et al. 2003 \\
\hline 1 & Universidad de Magallanes & $1997-2001$ & 6 & Área y Parque marino & Gibbons et al. 2003 \\
\hline $\mathrm{m}$ & Expedición CIMAR Fiordos 3 & 1998 & 2 & Canal Jerónimo, islas Charles & Antezana et al. 2002 \\
\hline $\mathrm{n}$ & Expedición CIMAR Fiordos 3 & 1998 & 1 & Canal Jerónimo & Ramírez 2005 \\
\hline$\tilde{n}$ & Expedición CIMAR Fiordos 3 & 1998 & 2 & Canal Jerónimo, islas Charles & Mujica \& Villablanca 2003 \\
\hline o & Expedición CIMAR Fiordos 3 & 1998 & 2 & Canal Jerónimo, islas Charles & Palma \& Aravena 2001 \\
\hline $\mathrm{p}$ & Expedición CIMAR Fiordos 3 & 1998 & 2 & Canal Jerónimo, islas Charles & Bernal \& Balbontín 2003 \\
\hline q & Universidad de Magallanes & 2001 & 5 & Área y Parque marino & Venegas et al. 2002 \\
\hline $\mathrm{r}$ & Expedición Chonos & 2003 & 1 & Seno Ballena & Valle-Levinson et al. 2006 \\
\hline $\mathrm{s}$ & Fundación CEQUA & $2003-2005$ & 6 & Área y Parque marino & Acevedo et al. 2006 \\
\hline $\mathrm{t}$ & Fundación CEQUA & $2003-2005$ & 6 & Área y Parque marino & Acevedo $2005^{4}$ \\
\hline $\mathrm{u}$ & Fundación CEQUA & 2007 & 10 & Área y Parque marino & CEQUA 2007a ${ }^{1}$ \\
\hline $\mathrm{v}$ & Centro Ecosistemas Patagonia & 2007 & 1 & Seno Ballena & Torres et al. 2011 \\
\hline $\mathrm{w}$ & Fundación CEQUA & 2007 & 6 & Área y Parque marino & CEQUA $2007 b^{2}$ \\
\hline $\mathrm{x}$ & Fundación Biomar & 2011 & 6 & Área y Parque marino & Aguayo-Lobo et al. 2011 \\
\hline y & Fundación Biomar & 2012 & 3 & C. Jerónimo, Carlos III, paso Shag & Presente Estudio \\
\hline
\end{tabular}

$75 \mathrm{~m}$ ) tendería a fluir hacia el océano, presentando baja salinidad y temperatura, alta concentración de oxígeno disuelto, alto $\mathrm{pH}$ y baja concentración de nutrientes. En cambio, la capa más profunda ( $>75$ $\mathrm{m})$ tendería a ingresar a los canales, presentaría mayor salinidad y temperatura, menor concentración de oxígeno, bajo $\mathrm{pH}$ y mayor concentración de nutrientes (Sievers, 2006).

Según un estudio realizado por la Fundación $\mathrm{CEQUA}^{2}$, sobre oceanografía del AMCP Francisco 

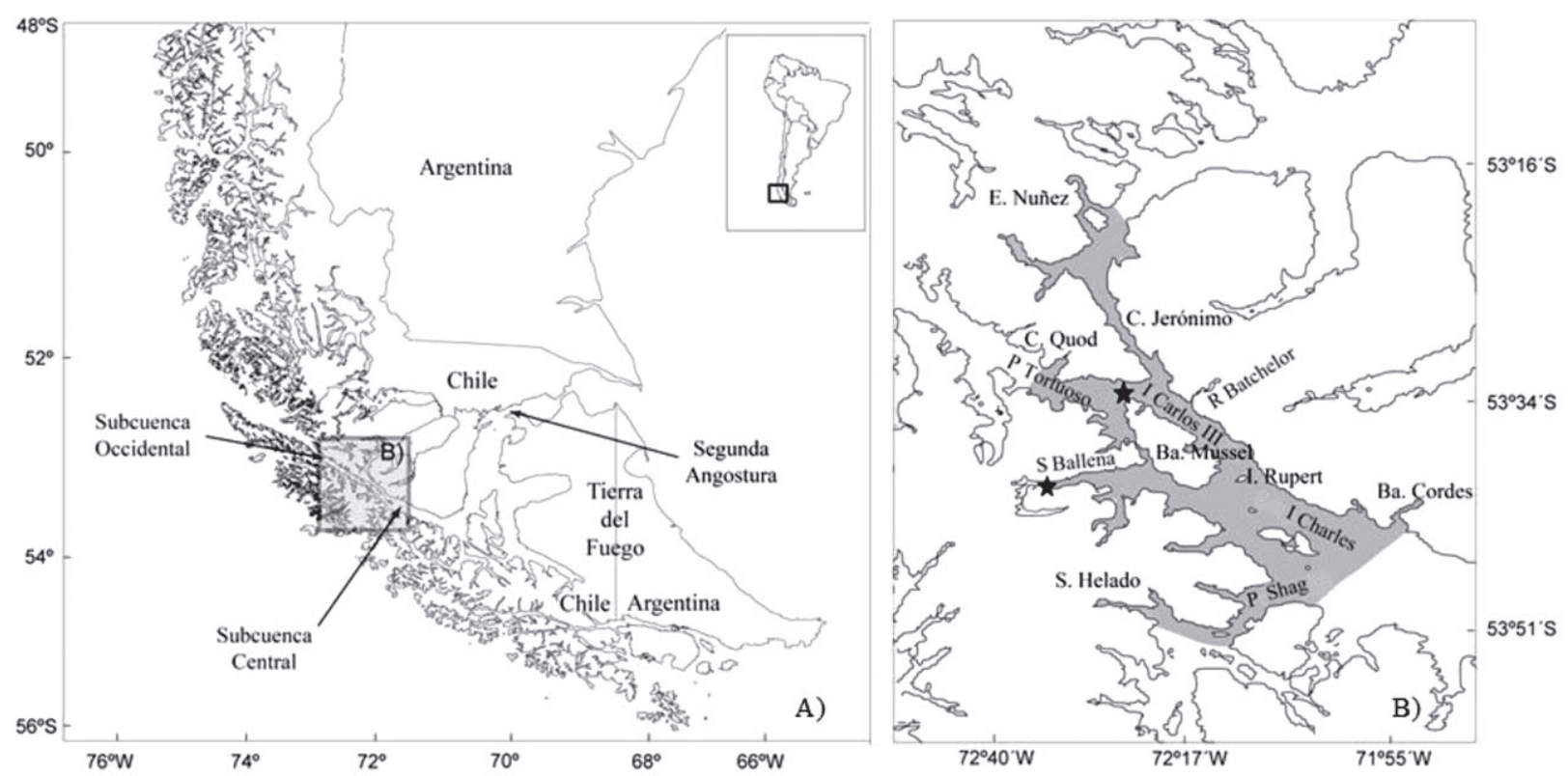

Fig. 1: A: Ubicación geográfica del Área Marina Costera Protegida Francisco Coloane (en gris) en el estrecho de Magallanes. B: AMCP Francisco Coloane. Estrellas negras señalan la ubicación aproximada de los umbrales presentes en el área.

Coloane, la circulación oceanográfica del área, estaría dominada por corrientes de marea, que son resultado de la interacción entre la marea del Atlántico y la del Pacífico. En el AMCP, las corrientes en llenante fluyen hacia el oeste y en vaciante hacia el este, alcanzando valores del orden de $60 \mathrm{~cm} \mathrm{~s}^{-1}$, desde superficie a fondo. El mismo estudio señala que en el sector de unión entre canal Jerónimo y el estrecho de Magallanes, el flujo proveniente del canal produce un giro ciclónico, que alcanza valores mayores a $60 \mathrm{~cm}$ $\mathrm{s}^{-1}$. Este giro en superficie se encuentra en el sector central del estrecho y en el fondo se desplaza hacia el norte.

En el AMCP Francisco Coloane, además del umbral localizado a $100 \mathrm{~m}$ de profundidad al oeste de isla Carlos III, se ha señalado la presencia de un umbral superficial a $3 \mathrm{~m}$ de profundidad en seno Ballena (Fig. 1), el cual correspondería a la sección expuesta y semihundida de una morrena terminal $\left(\mathrm{CEQUA}^{2}\right)$. Este umbral bloquea el paso de agua salada proveniente del estrecho hacia el interior del seno donde se ubica el glaciar. Sin embargo, en fases de marea alta, el agua salada sobrepasa el umbral produciendo surgencia de aguas ricas en nutrientes. Por otro lado, en marea baja, el agua llegaría solo hasta el umbral y las aguas del glaciar y de ríos cubrirían la zona entre el umbral y el glaciar (Valle-Levinson et al., 2006; Torres et al., 2011).

Temperatura y salinidad de las aguas del AMCP Francisco Coloane

Durante la prospección realizada en mayo de 2012 en el área de estudio, mes en el cual no se encontró información publicada sobre temperatura y salinidad en el AMCP, se registraron valores promedios de $7,7^{\circ} \mathrm{C}$ y 30,2 PSU en canal Jerónimo, $8,0^{\circ} \mathrm{C}$ y 30,8 PSU en Carlos III y $8,0^{\circ} \mathrm{C}$ y 30,9 PSU en paso Shag (Tabla 2).

La información publicada sobre temperatura superficial (<50 m profundidad) en el AMCP Francisco Coloane, señaló variaciones entre 2,0 ${ }^{\circ} \mathrm{C}$ en invierno y $9,0{ }^{\circ} \mathrm{C}$ en otoño (informados en canal Jerónimo). Se encontraron promedios de 2,5 ${ }^{\circ} \mathrm{C}$ en invierno y $8,2{ }^{\circ} \mathrm{C}$ en otoño (Tabla 2). Por su parte, la información de salinidad superficial señaló valores entre 23 PSU en verano en las cercanías del glaciar de seno Ballena, y 30,9 PSU en otoño 
Tabla 2: Temperatura $\left({ }^{\circ} \mathrm{C}\right)$ y Salinidad (PSU) registradas en el AMCP Francisco Coloane entre 1989 y 2012. (Referencias indicadas por letras).

\begin{tabular}{|c|c|c|c|c|c|c|c|c|c|c|c|c|}
\hline & \multicolumn{2}{|c|}{ Abril } & \multicolumn{2}{|c|}{ Mayo } & \multicolumn{2}{|l|}{ Julio } & \multicolumn{2}{|c|}{ Octubre } & \multicolumn{2}{|c|}{ Noviembre } & \multicolumn{2}{|c|}{ Diciembre } \\
\hline & $\mathrm{T}$ & $\mathrm{S}$ & $\mathrm{T}$ & S & $\mathrm{T}$ & $S$ & $\mathrm{~T}$ & S & $\mathrm{T}$ & S & $\mathrm{T}$ & S \\
\hline Canal Jerónimo & $8,0-9,0$ w & $29,2-30,8^{w}$ & $7,7^{\text {y }}$ & $30,2^{\text {y }}$ & $0-3,0^{d}$ & & & & & & & \\
\hline Carlos III & $8,5^{w}$ & $29,8-30,0^{w}$ & $8,0^{y}$ & $30,8^{y}$ & & & $6,2^{\mathrm{s}}$ & $30,8^{\mathrm{g}}$ & $7,2^{\text {a }}$ & & & \\
\hline Carlos III & & & & & & & & & $7,4-8,2^{v}$ & $, 4-30,5^{w}$ & & \\
\hline Paso David & $8,5^{w}$ & $29,4^{w}$ & & & & & & & & & & \\
\hline Entrada S. Ballena & $<8,0$ w & $29,8^{w}$ & & & & & & & & & & \\
\hline Seno Ballena & $7,5^{w}$ & $27,0-28,0$ w & & & & & & & & & $7,5-7,8^{w}$ & $29,0^{w}$ \\
\hline Interior S. Ballena & & & & & & & & & & & $5,9-7,7 \times 2$ & $3.0-25,0^{\vee}$ \\
\hline Islas Charles & $8,0^{w}$ & 30,0 w & & & & & $7,0-7,5^{i}$ & $30,5^{j}$ & & & & \\
\hline Islas Charles & & & & & & & $6,2^{j}$ & $30,8^{j}$ & & & & \\
\hline Paso Shag & & & $8,0^{y}$ & $30,9^{y}$ & & & & & & & & \\
\hline Promedio & 8,2 & 29,4 & 7,9 & 30,6 & 2,5 & & 6,5 & 30,7 & 7,5 & 30,0 & 7,2 & 26,5 \\
\hline
\end{tabular}

en paso Shag (boca canal Bárbara). Los promedios de salinidad encontrados fueron de 26,5 PSU en primavera y 30,7 PSU a comienzos de la misma estación.

Se ha señalado que la temperatura y salinidad de las aguas del AMCP, se caracterizan por ser bastante homogéneas tanto horizontal como verticalmente en los $50 \mathrm{~m}$ superficiales, durante noviembre y abril $\left(\mathrm{CEQUA}^{2}\right)$. Esta estructura térmica casi homogénea sería generada por fuertes vientos y corrientes de marea, las cuales son características frecuentes en la zona de estudio (Sievers, 2006). Al comparar los valores de temperatura y salinidad medidos en este estudio con la información previamente publicada, se observa una drástica diferencia estacional principalmente en la temperatura superficial de las aguas del AMCP. Los valores menores estarían asociados a descargas de ríos y/o derretimiento de hielo glaciar, destacando el glaciar en seno Ballena (CEQUA ${ }^{2}$; Sievers, 2006). Situación similar se ha registrado en la región, en seno Gallegos (Vásquez, et al. 2012), y en general en los canales del sur de Chile (Pickard, 1973).
Oxígeno disuelto y pH de las aguas del AMCP Francisco Coloane

La información publicada sobre oxígeno disuelto en las aguas del área de estudio, señala para las aguas superficiales de islas Charles 6,5 ml. $\mathrm{L}^{-1}$ (290 $\mu$ moles $\mathrm{Kg}^{-1}$ ) durante octubre (Valdenegro \& Silva, 2003), manteniendo valores similares en noviembre (280-300 umoles $\mathrm{Kg}^{-1}$ ), aunque ligeramente mayores en la capa superficial $(<5 \mathrm{~m})\left(\mathrm{CEQUA}^{2}\right)$. En diciembre, Torres et al. (2011) señaló entre 300 y 330 umoles $\mathrm{Kg}^{-1}$ de oxígeno en aguas superficiales de seno Ballena. Entre el umbral presente en esa zona y el estrecho, la concentración fue de 300 umoles $\mathrm{Kg}^{-1}$, en el umbral fue de $310 \mu$ moles $\mathrm{Kg}^{-1}$, y a orillas del glaciar alcanzó $330 \mu$ moles $\mathrm{Kg}^{-1}$ (Fig. 2A).

En cuanto a los valores de $\mathrm{pH}$ publicados, variaron entre 7,8 y 8,1 unidades en las aguas superficiales del AMCP. Información más localizada señala que las aguas de islas Charles presentan 7,8 unidades de $\mathrm{pH}$ en los primeros $30 \mathrm{~m}$ y 7,7 unidades a mayor profundidad en octubre (Valdenegro \& Silva, 2003), siendo ligeramente más básicos en los primeros $30 \mathrm{~m}$ durante noviembre (CEQUA $\left.{ }^{2}\right)$. No

\footnotetext{
${ }^{2}$ CEQUAb. 2007. Análisis y Diagnóstico de las principales características oceanográficas del Área Marina Costera Protegida Francisco Coloane. Informe Final. Programa de las Naciones Unidas para el Desarrollo, Comisión Nacional del Medio Ambiente. 136 Págs.
} 


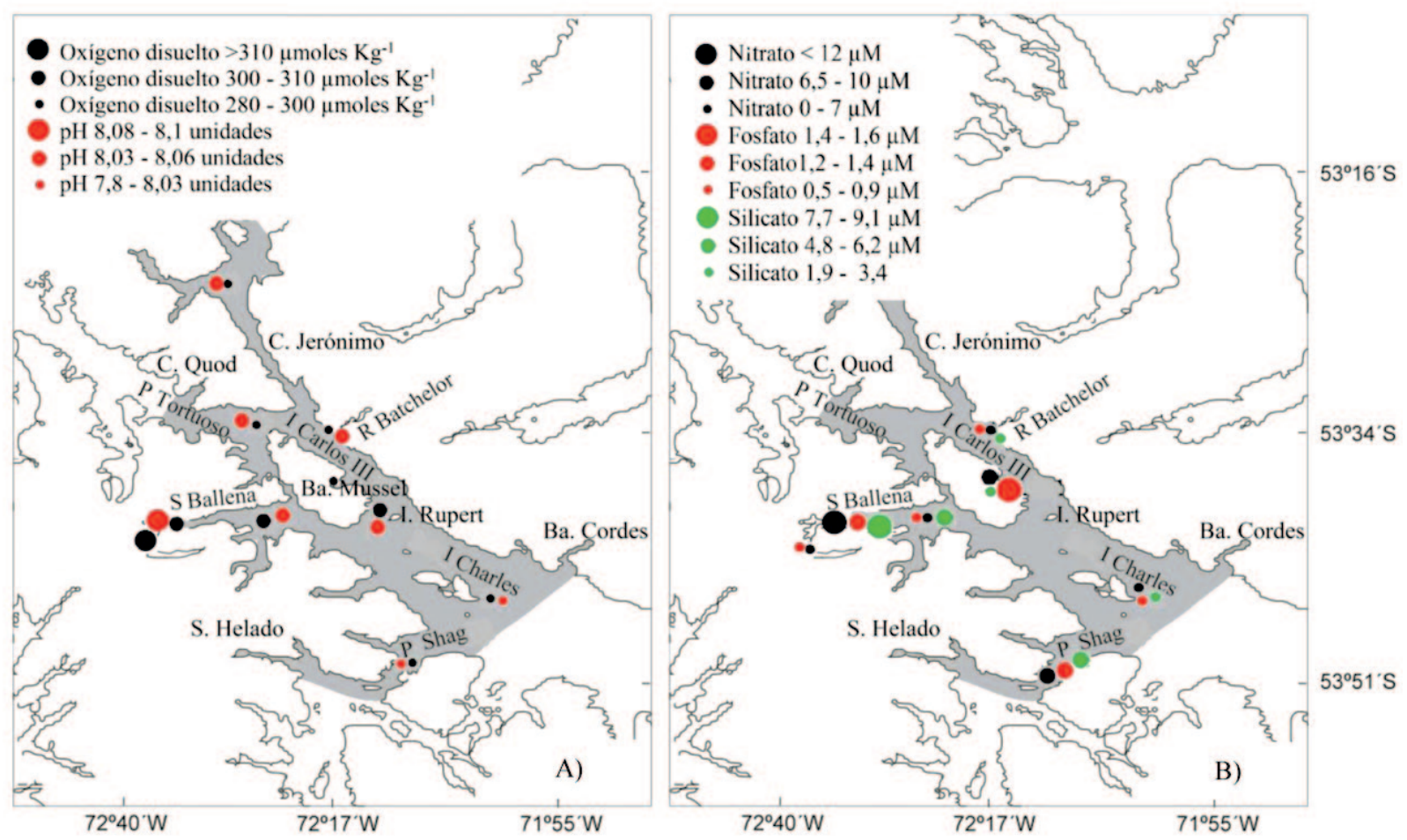

Fig. 2: A: Concentración de oxígeno disuelto y pH registrados en primavera en el AMCP Francisco Coloane. B: Niveles de nutrientes registrados en primavera en el AMCP Francisco Coloane.

obstante, las aguas más oxigenadas y con mayor $\mathrm{pH}$ se encontraron en noviembre en seno Ballena (320-340 $\mu$ moles $\mathrm{Kg}^{-1}$ y 8,11 unidades) y los menores valores en canal Jerónimo durante el mismo mes (280-290 umoles $\mathrm{Kg}^{-1}$ y 8,03 unidades) (CEQUA ${ }^{2}$; Torres et al., 2011) (Fig. 2A). Los altos valores de oxígeno y pH encontrados en las aguas superficiales del AMCP, se deberían al intercambio de oxígeno océano-atmósfera y a procesos fotosintéticos, los cuales producen aumento de oxígeno, disminución del anhídrido carbónico, y por tanto el aumento de $\mathrm{pH}$ (Silva, 2006). Los mayores valores de oxígeno y $\mathrm{pH}$ registrados en las cercanías del glaciar, se deberían principalmente a la temperatura del agua en esa zona.

Nutrientes de las aguas del AMCP Francisco Coloane

En relación a los nutrientes, Valdenegro \& Silva (2003), informan que las aguas de islas Charles, presentan valores de nitrato superficial de 6,5 a 8,0 $\mu \mathrm{M}$ y de fosfato de 0,7 a $0,9 \mu \mathrm{M}$ en octubre, señalando que bajo los $100 \mathrm{~m}$ el nitrato sería de $8,0 \mu \mathrm{M}$ y el fosfato superior a 1,0 $\mu \mathrm{M}$. También se ha informado que en noviembre, los niveles promedio de nitrato, fosfato y silicato en los $30 \mathrm{~m}$ superficiales, serían de $6 \pm 2 \mu \mathrm{M} ; 1,1 \pm 0,5 \mu \mathrm{M}$ y $4 \pm 3 \mu \mathrm{M}$ respectivamente, $\mathrm{y}$ que los valores menores estarían al interior de seno Ballena $\left(\mathrm{CEQUA}^{2}\right)$. Torres et al. (2011) registraron en las aguas entre el estrecho y el umbral de seno Ballena valores superficiales de nitrato entre 7 y 10 $\mu \mathrm{M}, \mathrm{y}$ de silicato entre 2 y $4 \mu \mathrm{M}$ en diciembre. Pasado el umbral, la concentración superficial de nitrato fluctuó entre 6 y $12 \mu \mathrm{M}$ y la de silicato se mantuvo entre 2 y $4 \mu \mathrm{M}$. A orillas del glaciar se encontraron valores de nitrato entre 0 y $7 \mu \mathrm{M}$ y de $1 \mu \mathrm{M}$ de silicato (Fig. 2B). Según los mismos autores, en marea alta, cuando se produce surgencia de aguas profundas, el agua superficial de seno Ballena queda enriquecida en nitrato y con bajo silicato. En cambio, en marea baja, las aguas superficiales presentan altos valores de silicato y bajos de nitrato. 
Tabla 3: Abundancia (células $\left.\mathrm{L}^{-1}\right)$ y biomasa fitoplanctónica $\left(\mathrm{mg} \mathrm{m}^{-3}\right)$ registradas en el AMCP Francisco Coloane entre 1991 y 2007. (Referencias indicadas por letras) * señala biomasa fitoplanctónica medida en $\mathrm{mg} \mathrm{m}^{-2}$.

\begin{tabular}{|c|c|c|c|c|c|c|c|c|c|c|c|c|}
\hline & \multicolumn{2}{|c|}{ Febrero } & \multicolumn{2}{|c|}{ Marzo } & \multicolumn{2}{|c|}{ Abril } & \multicolumn{2}{|c|}{ Octubre } & \multicolumn{2}{|c|}{ Noviembre } & \multicolumn{2}{|c|}{ Diciembre } \\
\hline & Abundancia & Biomasa & Abundancia & Biomasa & Abundancia & Biomasa & Abundancia & Biomasa & Abundancia & Biomasa & Abundancia & Biomasa \\
\hline Canal Jerónimo & & & & & & & $>1.000 .000^{\mathrm{k}}$ & $4-16^{m}$ & & & & \\
\hline Carlos III & $2,6-6,5 \times 10^{6} \mathrm{f}$ & $1,3^{\mathrm{e}}$ & $2,6-6.5 \times 10^{6 \mathrm{f}}$ & $1,3^{\mathrm{e}}$ & & $* 14,1^{\mathbf{h}}$ & & & & & & \\
\hline Carlos III & & & & $* 14,1^{\mathrm{h}}$ & & & & & & & & \\
\hline Ba. Mussel & & & & & & & & & & $>2^{w}$ & & \\
\hline Exterior S. Ballena & & & & & & & & & & & & $1-3^{v}$ \\
\hline Seno Ballena & & & & & & & & & & & & $0-3^{v}$ \\
\hline Interior S. Ballena & & & & & & & & & & $>2^{w}$ & & $0-8^{v}$ \\
\hline Islas Charles & & & & $* 16,6{ }^{\mathrm{h}}$ & & $* 16,6^{\mathrm{h}}$ & & $0-2^{m}$ & & & & \\
\hline Islas Charles & & & & & & & & $1,5^{\mathrm{g}}$ & & & & \\
\hline
\end{tabular}

Los niveles de nutrientes superficiales en el AMCP se encontrarían dentro del rango de valores registrados en seno Gallegos en la región de Magallanes (Vásquez, 2012), y serían menores a los señalados en los fiordos de la región de los Lagos y en Aysén (> $2 \mu \mathrm{M}$ de fosfato $y>20 \mu \mathrm{M}$ de nitrato) (Silva, 2006). En general los niveles promedio de nutrientes, indican que la concentración en la capa superficial no es alta, con excepción del fosfato, en el cual valores mayores a $2 \mu \mathrm{M}$ son consideradas como concentraciones altas. Estos valores son esperados debido a la fotosíntesis que se produce en la capa superficial, la cual disminuye los niveles, y a la influencia de agua dulce con baja concentración de nutrientes (Silva, 2006).

Composición y abundancia del fitoplancton en el AMCP Francisco Coloane

De acuerdo con Iriarte et al. (1993) y Antezana (1999), en primavera el fitoplancton del AMCP se caracteriza por presentar cadenas tróficas cortas formadas por grandes diatomeas (>20 $\mu \mathrm{m})$, $y$ en verano, el fitoplancton es dominado por pico $(0.2-2 \mu \mathrm{m})$ y nanofitoplancton $(2-20 \mu \mathrm{m})$, con una red trófica compleja mediada por un bucle bacteriano.

Para el AMCP se informó 43 taxa de fitoplancton, conformado por 27 especies de diatomeas, 12 dinoflagelados, 2 silicoflagelados y 2 ciliados (CEQUA ${ }^{2}$ ). Se registró el 32\% de las taxa señaladas en Magallanes, coincidiendo con el predominio de diatomeas. En estudios realizados durante el mes de octubre se señala alta diversidad de especies en islas Charles (Avaria et al., 2003) y baja diversidad en canal Jerónimo, pero con altas concentraciones de células $\left(>1 \times 10^{6}\right.$ células $\left.\mathrm{L}^{-1}\right)$ en los primeros $30 \mathrm{~m}$ (Ramírez, 2005; Tabla 3; Fig. 3A). En noviembre, al exterior de seno Ballena y en canal Jerónimo, se registró la mayor cantidad de taxa (11 y 13 especies, respectivamente). Además, altos valores de células se registraron en los $30 \mathrm{~m}$ superficiales frente a río Batchelor $\left(\mathrm{CEQUA}^{2}\right)$. Durante el mismo mes, Uribe (1991) señaló gran diversidad de diatomeas en Carlos III (> 20 especies). En febrero y marzo, se encontró alta concentración de células en la subcuenca occidental, incluyendo el noroeste de Carlos III, con valores entre 2.6 a $6.5 \times 10^{6}$ células $\mathrm{L}^{-1}$ (Zingone et al., 2011; Tabla 3).

\section{Biomasa fitoplanctónica en el AMCP Francisco Coloane}

En relación a la concentración de Clorofila-a en el AMCP, en las aguas de islas Charles se registraron valores entre 0 y $1,5 \mathrm{ug} / 1\left(1,5 \mathrm{mg} \mathrm{m}^{-3}\right) \mathrm{y}$ en canal Jerónimo valores entre 4-16 $\mathrm{mg} \mathrm{m}^{-3}$ en los 50 m superiores en octubre (Arntz \& Gorny, 1996; Antezana et al., 2002; Ramírez, 2005; Tabla 3; Fig. 3A). Según señala el informe de la Fundación CEQUA $^{2}$ la concentración de Clorofila-a en el área de estudio es moderada 0,4 a $2{\mu \mathrm{gl}^{-1}}^{-1}(0,4$ a $2 \mathrm{mg}$ $\mathrm{m}^{-3}$ ), y al interior de seno Ballena y en bahía Mussel (Carlos III) sería alta $\left(>2 \mu \mathrm{gl}^{-1}\right)\left(>2 \mathrm{mg} \mathrm{m}^{-3}\right)$ durante noviembre. En diciembre, en las aguas entre el umbral de seno Ballena y el estrecho, la concentración fluctuó entre 1-3 $\mathrm{mg} \mathrm{m}^{-3}$, y en el umbral se encontraron valores entre $0-3 \mathrm{mg} \mathrm{m}^{-3}$. Al interior de este seno las concentraciones de Clorofila-a variaron entre 0-8 $\mathrm{mg} \mathrm{m}^{-3} \mathrm{y}$ a orillas del glaciar fluctuaron entre $0-2 \mathrm{mg}$ $\mathrm{m}^{-3}$ (Torres et al., 2011; Fig. 3A). Durante febrero 


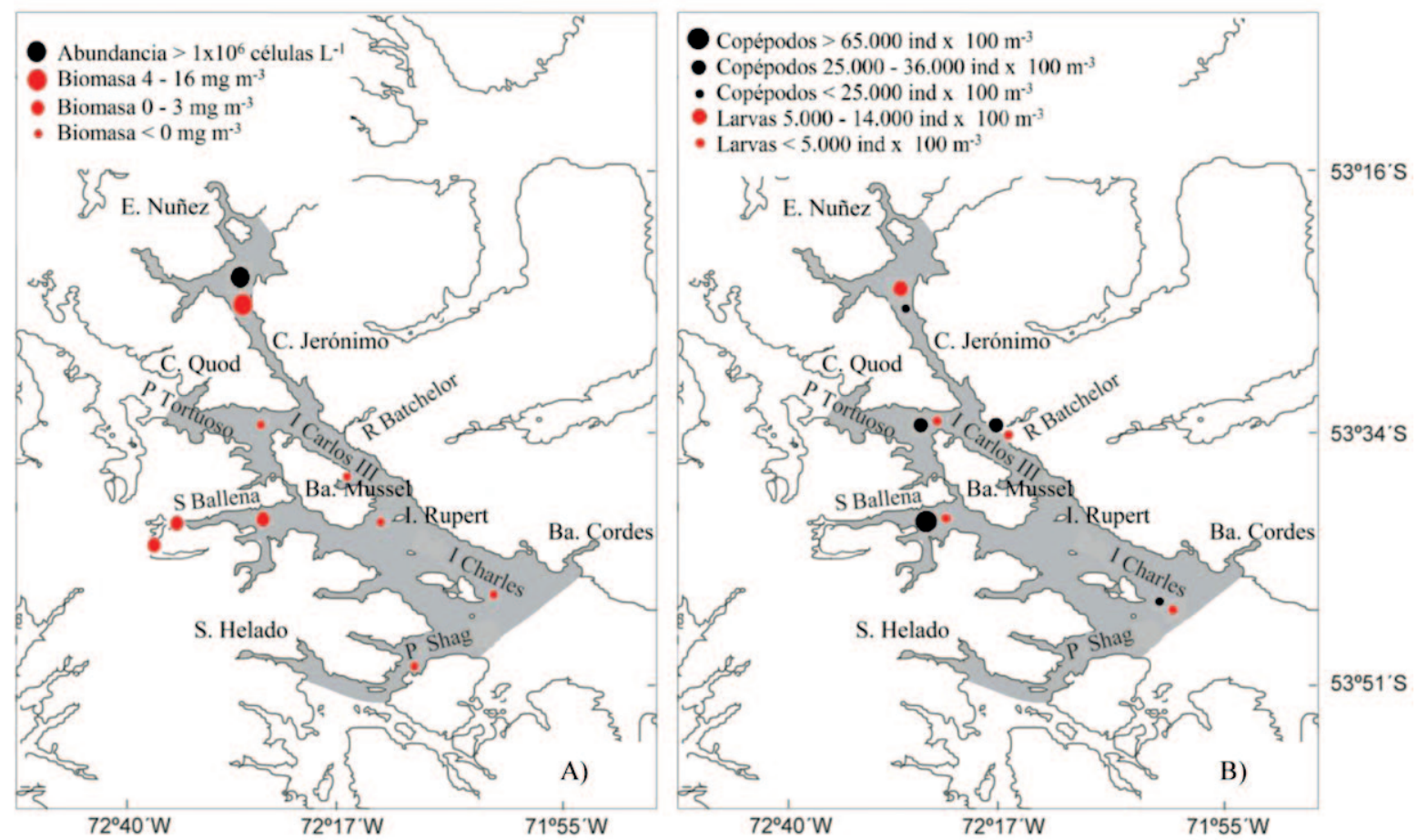

Fig. 3: A: Abundancia y biomasa fitoplanctónica registrada en primavera en el AMCP Francisco Coloane. B: Abundancia de copépodos y larvas registrada en primavera en el AMCP Francisco Coloane.

y marzo, Carrada et al., (1994) señalaron para la subcuenca occidental, incluyendo el noroeste de Carlos III, una concentración promedio de Clorofila-a de 1,3 $\mu \mathrm{g} \mathrm{dm}{ }^{-3}\left(1,3 \mathrm{mg} \mathrm{m}^{-3}\right)$, señalando además que en esa subcuenca los valores disminuyen notablemente $(0,6$ $\left.\mu \mathrm{g} \mathrm{dm}^{-3}\right)\left(0,6 \mathrm{mg} \mathrm{m}^{-3}\right)$. En marzo y abril, se registraron concentraciones de $14,1 \mathrm{mg} \mathrm{m}^{-2}\left(14,1 \mathrm{mg} \mathrm{m}^{-3}\right)$ al noroeste de Carlos III y de $16,6 \mathrm{mg} \mathrm{m}^{-2}(16,6 \mathrm{mg}$ $\mathrm{m}^{-3}$ ) en islas Charles (Saggiomo et al., 2011; Tabla 3).

En general, las concentraciones de Clorofila-a en el AMCP fluctuarían entre 0,4 y $16 \mathrm{mg} \mathrm{m}^{-3}$, registrándose los mayores valores en primavera, estas concentraciones son más altas que las registradas en el estrecho de Magallanes (promedio $=0,32 \mathrm{mg} \mathrm{m}^{-3}$ ) durante la misma estación (Saggiomo et al., 2011).

Ramírez (2005), señaló que los sistemas productivos poseen baja diversidad de especies y alta biomasa. Sin embargo, según la revisión presentada, la diversidad, abundancia y biomasa fitoplanctónica no es igual en todas las aguas que componen el AMCP y presentarían variaciones a lo largo del año, como canal Jerónimo que en octubre tendría baja diversidad de especies, alta abundancia de células y alta biomasa (Antezana et al., 2002), $y$ en noviembre, la diversidad aumenta $\left(\mathrm{CEQUA}^{2}\right)$, disminuyendo probablemente la biomasa.

En cuanto a floraciones algales nocivas, cabe señalarse que al interior de seno Ballena se registró un dinoflagelado potencialmente toxico, Alexandrium ostenfeldii (Paulsen, 1904), con 16.500 células $\mathrm{ml}^{-1}\left(16,5\right.$ células $\left.\mathrm{L}^{-1}\right)$ a los $5 \mathrm{~m}$ de profundidad durante noviembre, pudiendo en algún momento formar floraciones en el AMCP. En islas Charles se registró Alexandrium catenella (Whedon \& Kofoid, Balech, 1985) pero en una abundancia muy baja $\left(0,5\right.$ células $\left.\mathrm{L}^{-1}\right)\left(\mathrm{CEQUA}^{2}\right)$.

Según Uribe (1991), Antezana et al. (1992) y Palma (2006), tanto el fitoplancton como el zooplancton del AMCP están conformados por una mezcla de especies de diferentes regiones biogeográficas, ya sea por especies antárticas, 
Tabla 4: Presencia y abundancia de zooplancton en primavera en el AMCP Francisco Coloane entre 1989 y 2007. (Referencias indicadas por letras).

\begin{tabular}{|c|c|c|}
\hline Lugar AMCP & Abundancia & Organismos Zooplanctónicos \\
\hline C. Jerónimo ñ,v & Alta & Copépodos, cladóceros, larvas de decápodos, eufáusidos, quetognatos \\
\hline Carlos III c,v & Alta & Copépodos, cladóceros, larvas de briozoos, tintínidos \\
\hline Río Batchelor v & Baja & Copépodos, cladóceros \\
\hline Islas Charles $\tilde{n}, \mathrm{o}, \mathrm{v}$ & Baja & $\begin{array}{c}\text { Copépodos, larvas de decápodos y de briozoos, eufáusidos, } \\
\text { quetognatos, sifonóforos }\end{array}$ \\
\hline Seno Ballena v & Alta & Copépodos, eufáusidos, larvas de eufáusidos, ostrácodos \\
\hline
\end{tabular}

subantárticas y cosmopolitas, y en el caso del fitoplancton hasta por especies de aguas temperadas. La mayoría de estas taxa penetran al estrecho a través de la boca occidental donde existe mayor profundidad. Las aguas del AMCP no tendrían especies endémicas, debido al constante intercambio de aguas oceánicas con aguas estuarinas, lo que causa circulación de masas de agua que provocan la entrada y salida de las especies, y serían pocas las taxa que podrían colonizar esta zona debido a esta alta variabilidad oceanográfica.

\section{Zooplancton en el AMCP Francisco Coloane}

Durante primavera la comunidad zooplanctónica del AMCP, está compuesta principalmente por copépodos y larvas meroplanctónicas (Antezana, 1999; CEQUA²: Fig. 3B).

Según Mujica \& Villablanca (2003) y CEQUA², canal Jerónimo durante octubre y noviembre, se caracteriza por presentar alta abundancia de copépodos, cladóceros y larvas de decápodos. Las abundancias fluctuarían entre 4.915 y 13.359 larvas $/ 1000 \mathrm{~m}^{3}$ de decápodos, destacando el infraorden Anomura y Brachyura. Palma \& Aravena (2001) registraron la presencia de eufáusidos (Euphausia vallentini, John, 1936) y quetognatos (Sagitta tasmanica, Thomson, 1947) en menor abundancia (64-365 ind $\left./ 1000 \mathrm{~m}^{3}\right)$, y señalaron que la biomasa zooplanctónica en octubre fue entre 32 y 64 $\mathrm{ml} / 1000 \mathrm{~m}^{3}$. Esta biomasa coincide con la alta densidad fitoplanctónica registrada durante ese mes en canal Jerónimo, lo cual indica que habría una asociación entre las proliferaciones primaverales de fitoplancton y la abundancia zooplanctónica. En noviembre, además de copépodos (24.918 ind $\mathrm{x}$ $100 \mathrm{~m}^{-3}$ ), destacaron en abundancia los cladóceros con 3.209 ind x $100 \mathrm{~m}^{-3}$ (CEQUA ${ }^{2}$ ) (Tabla 4).

En islas Charles el zooplancton está representado principalmente por copépodos y larvas (Arntz \& Gorny, 1996; Fig. 3B). En octubre habría baja abundancia de organismos, destacando los copépodos con 16.990 ind $\times 100 \mathrm{~m}^{-3}$, las larvas de eufáusidos con 471 ind $x 100 \mathrm{~m}^{-3} \mathrm{y}$ las de briozoos con 545 ind $x 100 \mathrm{~m}^{-3}\left(\mathrm{CEQUA}^{2}\right)$, en tanto que Mujica \& Villablanca (2003) señalan una abundancia de larvas de decápodos entre 1.809 a 4.914 larvas/1000 $\mathrm{m}^{3}$. La biomasa zooplanctónica en octubre fue baja (1-32 ml /1000m $\left.{ }^{3}\right)$. Se registró la presencia de E. vallentini y $S$. tasmanica con abundancias de 64 a 365 ind/1000 $\mathrm{m}^{3}$ y del sifonóforo Muggiaea atlantica (Cunningham, 1892) con una abundancia entre 1 y 64 ind $/ 1000 \mathrm{~m}^{3}$ (Palma \& Aravena, 2001). Hamamé \& Antezana (2010) señalaron la presencia de E. vallentini en aguas superficiales $(<200 \mathrm{~m}) \mathrm{de}$ esta zona en marzo y principios de abril.

En el noroeste de isla Carlos III, se registró alta abundancia de copépodos (35.631 ind x 100 $\mathrm{m}^{-3}$ ), cladóceros (20.928 ind $\left.\mathrm{x} 100 \mathrm{~m}^{-3}\right)$ y larvas de briozoos (2.696 ind $\mathrm{x} 100 \mathrm{~m}^{-3}$ ) en noviembre $\left(\mathrm{CEQUA}^{2}\right)$. Por otra parte, se informó en superficie alta abundancia de tintínidos $\left(15,6\right.$ ind $\left.\mathrm{dm}^{-3}\right)$, los cuales son constituyentes del microzooplancton (FondaUmani \& Monti, 1991). En la desembocadura de río Batchelor, habría baja abundancia de organismos en noviembre, registrándose copépodos (26.810 ind $\mathrm{x}$ $100 \mathrm{~m}^{-3}$ ) y cladóceros (1.312 ind x $100 \mathrm{~m}^{-3}$ ), y al exterior de seno Ballena destacaron los copépodos (69.216 ind x $100 \mathrm{~m} 3$ ), eufáusidos adultos (373 ind x $100 \mathrm{~m}^{-3}$ ), larvas (4.284 ind x $100 \mathrm{~m}^{-3}$ ), y ostrácodos 
(2.607 ind x $100 \mathrm{~m}^{-3}$ ) (CEQUA2 ${ }^{2}$; Tabla 4; Fig. 3B). Además, se registró alta biomasa de langostino de los canales (Munida gregaria, Fabricius, 1793), el cual forma densas agregaciones, y que junto con el krill (Euphausia vallentini), (Arntz \& Gorny, 1996; Schnack-Schiel \& Isla, 2005), tendrían una función pelago-bentónica, al generar un flujo constante de energía desde la superficie hacia el bentos, con la migración vertical que realizan y con sus fecas de gran tamaño, las cuales se hunden rápidamente y presentan alto valor nutritivo, ejerciendo una influencia positiva en la producción bentónica (Antezana, 1999; Schnack-Schiel \& Isla, 2005). La función de estas especies en los fiordos podría ser comparable a la acción del krill (Euphausia superba, Dana 1850) en la Antártica, debido a su abundancia (Antezana, 1999).

\section{Peces en el AMCP Francisco Coloane}

En el AMCP se han registrado más de 20 especies de peces, destacando la familia Nototheniidae del orden Perciformes, con los géneros Paranotothenia, Patagonothen y Dissostichus. Se han registrado en el área, la sardina fueguina (Sprattus fueguensis, Jenyns, 1842), el puye (Galaxias maculatus, Jenyns, 1842), el róbalo (Eleginops maclovinus, Cuvier, 1830), la merluza austral (Merluccius australis, Hutton, 1872), la merluza de cola (Macruronus magellanicus, Lönnberg 1907) y el congrio dorado (Genypterus blacodes, Forster 1801) (Pequeño, 2000; Bernal \& Balbontín, 2003; Pequeño, 2006; Aguayo-Lobo et al., 2011). Sin embargo, estudios de la biología y del papel ecológico que cumplen estas especies en el ecosistema son escasos (Pequeño et al., 1995; Pequeño, 2000).

Según Bernal \& Balbontín (2003) y Balbontín (2006) el estrecho de Magallanes presenta las condiciones favorables para el crecimiento y desarrollo de larvas de peces, destacando en abundancia las larvas de merluza de cola y de sardina fueguina en los $50 \mathrm{~m}$ superiores en primavera, siendo la sardina fueguina la especie con mayor dominancia larval en la región. En las aguas del AMCP, se encontró una abundancia de larvas de merluza de cola entre 5,97 a 35,33 larvas x $10 \mathrm{~m}^{2}$ en canal Jerónimo durante octubre, y en aguas adyacentes a islas Charles se registró una abundancia de larvas de chancharro (Sebastes capensis, Valenciennes, 1833) de 12,29 a 37,31 larvas x $10 \mathrm{~m}^{2}$ (Bernal \& Balbontín, 2003). La sardina fueguina es una especie pelágica costera que forma grandes cardúmenes y su tamaño adulto fluctúa entre los 14 y $18 \mathrm{~cm}$. Observaciones personales de los autores durante los meses de verano y otoño en el área de estudio, permiten informar que la especie es muy abundante, sirviendo de alimento no solo a la ballena jorobada, sino que también a los lobos marinos comunes y finos, y a numerosas especies de aves. Por tanto, se estima que la sardina podría jugar un papel clave en el ecosistema pelágico del área, evidenciándose la necesidad de realizar estudios sobre su biología y abundancia.

Mamíferos y aves marinas en el AMCP Francisco Coloane

Desde el año 2000 se han registrado 13 especies de mamíferos marinos y 32 especies de aves marinas y/o costeras en el AMCP Francisco Coloane. Entre los cetáceos destaca la ballena jorobada, especie que migra anualmente a estas aguas para alimentarse, y de manera ocasional, la ballena boba (Balaenoptera borealis, Lesson, 1828), la ballena minke antártica (Balaenoptera bonaerensis, Burmeister 1867), la orca (Orcinus orca, Linnaeus, 1758), el delfín austral (Lagenorynchus australis, Peale, 1848), el delfín chileno (Cephalorhynchus eutropia, Gray, 1846), la tonina overa (Cephalorhynchus commersonii, Lacépede, 1804) y el tursión (tursiops truncatus, Montagu, 1821) (Aguayo-Lobo et al., 1998; Gibbons et al., 2003; Acevedo, 2005; Acevedo et al., 2006; CEQUA ${ }^{1}$; Olavarría et al., 2010). El huillín (Lontra provocax, Thomas,1908), el lobo marino común, el lobo fino austral, estarían presentes la mayor parte del año, mientras que la foca elefante (Mirounga leonina, Linnaeus, 1758) y la foca leopardo (Hydrurga leptonyx, Blainville, 1820) serían visitantes ocasionales (Venegas et al.,2002;

\footnotetext{
${ }^{3}$ Acevedo, J. 2005. Distribución, filopatría, residencia e identidad poblacional de la ballena jorobada, Megaptera novaeangliae, que se alimentan en las aguas del estrecho de Magallanes, Chile. Tesis para optar a la Maestría en Ciencias. Facultad de Ciencias. Universidad de Magallanes.144 Págs. ${ }^{4}$ Haro, D. 2013. La ballena jorobada (Megaptera novaeangliae) y sus relaciones tróficas en el Área Marina Costera Protegida Francisco Coloane, estrecho de Magallanes. Tesis para optar a la Maestría en Ciencias. Facultad de Ciencias. Universidad de Magallanes.139 Págs.
} 
CEQUA $^{1}$; Aguayo-Lobo et al., 2011; Acevedo \& Martínez, 2013).

Entre las aves destacan el pingüino de Magallanes, el cormorán imperial (Phalacrocorax atriceps, King, 1828), el cormorán de las rocas (P. magellanicus, Gmelin, 1789), la skúa chilena (Catharacta chilensis, Bonaparte, 1857), el albatro de ceja negra (Thallasarche melanophris, Temminck 1828), el petrel plateado (Fulmarus glacialoides, Smith, 1840), el yunco de Magallanes (Pelecanoides magellani, Mathews, 1912), la fardela negra (Puffinus griseus, Gmelin, 1789), el petrel gigante (Macronectes giganteus, Gmelin, 1789) y el quetro no volador (Tachyeres pteneres, Forster, 1844) (CEQUA $\left.{ }^{1}\right)$.

En esta área, existen zonas de reproducción de dos colonias de lobo marino común, ubicadas en islote Rupert y en seno Helado, y apostaderos de lobo marino común y de lobo fino, los cuales han sido registrados en los meses de verano (Venegas et al., 2002). El AMCP es zona de reproducción del pingüino de Magallanes, del cormorán imperial y de las rocas, de la skúa chilena, de la gaviota común (Larus dominicanus, Lichtenstein 1823) y del gaviotín sudamericano (Sterna hirundinacea, Lesson 1831) (CEQUA $\left.{ }^{1}\right)$.

Estas aguas constituyen además, un área de alimentación y crianza para la ballena jorobada del Pacifico suroriental, la cual se concentra en la zona durante verano y otoño (Gibbons et al., 2003, Acevedo, 2005³ Acevedo et al., 2006) y se estaría alimentando principalmente de langostino de los canales y sardina fueguina (Haro, 2013) ${ }^{4}$.

\section{CONCLUSIONES}

Se informan por primera vez datos de temperatura y salinidad en la boca del canal Bárbara, y para el mes de mayo se informan además, valores de estos parámetros en canal Jerónimo e isla Carlos III.

En primavera destaca canal Jerónimo por su alta abundancia fito (diatomeas) y zooplanctónica (copépodos y larvas de decápodos). Esta última comunidad estaría dominada por pocas especies pero abundantes, como el langostino de los canales que tiende a formar densas agregaciones. En el componente íctico del zooplancton destacan las larvas de merluza de cola y sardina fueguina, que junto al langostino de los canales podrían sustentar las comunidades de vertebrados superiores, ya que los eufáusidos por alguna razón desconocida no son tan abundantes como se esperaría.

El AMCP presenta alta diversidad de aves y mamíferos marinos, la cual estaría sustentada por la productividad de las aguas, destacando especies emblemáticas como la ballena jorobada, dos especies de lobo marino y el pingüino de Magallanes.

Al terminar la revisión sobre las características oceanográficas y biológicas del ecosistema pelágico del AMCP Francisco Coloane, se estima haber cumplido de manera general el objetivo de este trabajo. En lo particular, se identificaron debilidades en el conocimiento de la comunidad bentónica del área de estudio, y en aspectos de oceanografía física como batimetría y circulación de masas de aguas. Se sugiere realizar estudios sistemáticos que abarquen la totalidad del Área Marina, y que se extiendan a los diferentes meses del año.

\section{AGRADECIMIENTOS}

Se agradece a la Fundación BIOMAR y a la tripulación de la M/N Forrest, por el apoyo logístico en la toma de datos en el mar. Al Dr. Cristian Rodrigo de INACH y al Sr. Emilio Mena de la Universidad Católica de Valparaíso, por el registro de datos de temperatura y salinidad en el área de estudio. A los doctores, Máximo Frangópulos de la Fundación CEQUA, Rodrigo Hucke-Gaete y Américo Montiel por sus comentarios y sugerencias al manuscrito. A las direcciones de la Fundación CEQUA y de INACH por las facilidades otorgadas para la realización de este manuscrito.

\section{LITERATURA CITADA}

Acevedo, J., Aguayo-Lobo, A., \& Pastene, L. (2006). Filopatría de la ballena jorobada (Megaptera novaeangliae, Borowski 1781), al área de alimentación del estrecho de Magallanes. Revista de Biología Marina y Oceanografía, 41, 11-19.

Acevedo, J., \& Martínez, F. (2013). Residence of Hydrurga leptonyx in Magallanes: a potential Subantarctic population that inhabit in Chile? Polar Biology, 36 (3), 453-456.

Aguayo-Lobo, A., Torres, D., \& Acevedo, J. (1998). Los Mamíferos Marinos de Chile: I. Cetácea. Serie Científica INACH, 48, 19-159.

Aguayo-Lobo, A., Acevedo, J., \& Cornejo, S. (2011). 
La ballena jorobada, Conservación en el Parque Marino Francisco Coloane. Santiago: Ocho Libros.

Andrade, S. (1991). Geomorfología costera y antecedentes oceanográficos físicos de la Región de Magallanes, Chile (48-56으). Anales Instituto Patagonia, 20 (1), 135-151.

Antezana, T., Guglielmo, L., \& Ghirardelli, E. (1992). Microbasins within the Strait of Magellan affecting zooplankton distribution. En: V. V. Gallardo, O. Ferretti \& H. I. Moyano (Eds.), Oceanografía en Antártica (pp. 453-458). Santiago: Ediciones documentadas.

Antezana, T. (1999). Plankton of Southern Chilean fjords: trends and linkages. Scientia Marina, 63 (1), 69-80.

Antezana, T., Giraldo, A., \& Hamamé, M. (2002). Clorofila y alimentación del zooplancton fraccionado por tamaño, en subcuencas del Sistema de Canales Magallánicos y fueguinos durante la primavera de 1998. Revista Ciencia y Tecnología del Mar, 25, 109-130.

Arntz, W.E., \& Gorny, M. (1996). Cruise report of the Joint Chilean-German-Italian Magellan "Victor Hensen" Campaign in 1994. Berichte zur Polarforschung, 190, 1-85.

Avaria, S., Cáceres, C., Castillo, P., \& Muñoz, P. (2003). Distribución del microfitoplancton marino en la zona Estrecho de Magallanes-Cabo de Hornos, Chile, en la primavera de 1998 (Crucero Cimar-3 Fiordos). Revista Ciencia y Tecnología del Mar, 26, 79-96.

Balbontín, F. (2006). Ictioplancton de los canales y fiordos australes. En: N. Silva, \& S. Palma (Eds.), Avances en el conocimiento oceanográfico de las aguas interiores Chilenas, Puerto Montt a cabo de Hornos (pp. 115-120). Valparaíso: Comité Oceanográfico Nacional.

Bernal, R., \& Balbontín, F. (2003). Distribución y abundancia de las larvas de peces desde el Estrecho de Magallanes hasta el Cabo de Hornos. Revista Ciencia y Tecnología del Mar, 26, 85-92.

Cabezas, A. (2006). Área Marina y Costera Protegida de Múltiples Usos Francisco Coloane. En: Ocho Libros (Eds.), Conservación de la biodiversidad de importancia mundial a lo largo de la costa chilena (pp. 136-139). Santiago: Salesianos S.A.

Carrada, G.C., Fabiano, M., Povero, P., \& Saggiomo,
V. (1994). Surface distribution of size-fractionated chlorophyll a and particulate organic matter in the strait of Magellan. Polar Biology, 14, 447-454.

Cornejo, S., \& Kush, A. (2006). Biodiversidad del Área Marina y Costera Protegida Francisco Coloane: Desafíos y Oportunidades. En: Ocho Libros (Eds.), Conservación de la biodiversidad de importancia mundial a lo largo de la costa chilena (pp. 142-146). Santiago: Salesianos S.A.

De Andrade, R. (2006). Introducción. En: Ocho Libros (Eds.), Conservación de la biodiversidad de importancia mundial a lo largo de la costa chilena (pp. 21-25). Santiago: Salesianos S.A.

Dudley, N. (2008). Directrices para la aplicación de las categorías de gestión de áreas protegidas. Gland: UICN.Fonda Umani, S. \& Monti, M. (1991). Microzooplankton populations in the Strait of Magellan. Bollettino di Oceanologia Teorica ed Applicata, 9 (2-3), 151-162.

Gibbons, J., Capella, J.C., \& Valladares, C. (2003). Rediscovery of a humpback whale (Megaptera novaeangliae) feeding ground in the Straits of Magellan, Chile. Journal of Cetacean Research and Management, 5 (2), 203-208.

Hamamé, M., \& Antezana, T. (2010). Vertical diel migration and feeding of Euphausia vallentini within southern Chilean fjords. Deep-Sea Research II, 57, 642-651.

Iriarte, J.L, Uribe, J.C., \& Valladares, C. (1993). Biomass of size-fractionated phytoplankton during the spring-summer season in the Southern Chile. Botánica Marina, 36, 443-450.

Mujica, A., \& Villablanca, R. (2003). Larvas de crustáceos decápodos en los fiordos y canales magallánicos. Revista Ciencia y Tecnología del Mar, 26, 73-84.

Olavarría, C., Acevedo, J., Vester, H. I., ZamoranoAbramson, J., Viddi, F.A., Gibbons, J., Newcombe, E., Capella, J., Hoelzel, A. R., Flores, M., Hucke-Gaete, R., \& Torres-Flórez, J. P. (2010). Southernmost distribution of common bottlenose dolphins (Tursiops truncatus) in the eastern South Pacific. Aquatic Mammals, 36 (3), 288-293.

Palma, S., \& Aravena, G. (2001). Distribución de quetognatos, eufáusidos y sifonóforos en la región Magallánica. Revista Ciencia y Tecnología del Mar, 24, 47-59.

Palma, S. (2006). Zooplankton distribution and 
abundance in the austral Chilean channels and fjords. En: N. Silva, \& S. Palma (Eds.): Avances en el conocimiento oceanográfico de las aguas interiores Chilenas, Puerto Montt a cabo de Hornos (pp. 107-113). Valparaíso: Comité Oceanográfico Nacional.

Panella, S., Michellato, A., Perdicaro, R., Magazzu, G., Decembrini, F., \& Scarazzato, P. (1991). A preliminary contribution to understanding the hydrological characteristics of the Strait of Magellan: Austral spring 1989. Bollettino di Oceanologia Teorica ed Applicata, 9 (2-3), 107-126.

Pequeño, G., Lamilla, J., Lloris, D., \& Rucabado, J. (1995). Comparación entre las ictiofaunas intermareales de los extremos austral y boreal de los canales Patagónicos. Revista de Biología Marina, 30, 155-177.

Pequeño, G. (2000). Peces del Crucero CIMAR 3 Fiordos, a los canales del sur de Magallanes (ca.55으), Chile. Revista Ciencia y Tecnología del Mar, 23, 83-94.

Pequeño, G. (2006). Peces litorales de los canales y fiordos australes. En: N. Silva, \& S. Palma (Eds.): Avances en el conocimiento oceanográfico de las aguas interiores Chilenas, Puerto Montt a cabo de Hornos. (pp. 129-132). Valparaíso: Comité Oceanográfico Nacional.

Pickard, G. L. (1973). Water structures in Chilean Fjords. In: R. Fraser (Eds.): Oceanography of the South Pacific. (pp. 95-104). Wellington: National Commission for UNESCO.

Ramírez, B. (2005). Distribución vertical de clorofila en los fiordos australes ubicados entre el estrecho de Magallanes y el cabo de Hornos (Chile). Revista Ciencia y Tecnología del Mar, 28 (2), 43-61.

Saggiomo, V., Santarpia, I., Saggiomo, M., Margiotta, F., \& Mangoni, O. (2011). Primary production processes and photosynthetic performance of a unique periantarctic ecosystem: the Strait of Magellan. Polar Biology, 34 (9), 1255-1267.

Schnack-Schiel, S.B., \& Isla, E. (2005). The role of zooplankton in the pelagic-benthic coupling of the Southern Ocean. Scientia Marina, 69 (II), 39-55.

Sievers, H. A. (2006). Temperatura y salinidad en canales y fiordos australes. En: N. Silva, \& S.
Palma (Eds.): Avances en el conocimiento oceanográfico de las aguas interiores Chilenas, Puerto Montt a cabo de Hornos. (pp. 31-36). Valparaíso: Comité Oceanográfico Nacional.

Silva, N. (2006). Oxígeno disuelto, pH y nutrientes en canales y fiordos australes. En: N. Silva, \& S. Palma (Eds.): Avances en el conocimiento oceanográfico de las aguas interiores Chilenas, Puerto Montt a cabo de Hornos. (pp. 37-43) Valparaíso: Comité Oceanográfico Nacional.

Torres, R., Frangópulos, M., Hamamé, M., Montecino, V., Maureira, C., Pizarro, G., Reid, B., ValleLevinson, A., \& Blanco, J. (2011). Nitrate to silicate ratio variability and the composition of micro-phytoplankton blooms in the inner-fjord of Seno Ballena (Strait of Magellan, $54^{\circ} \mathrm{S}$ ). Continental Shelf Research, 31, 244-253.

Uribe, J. C. (1991). Net-phytoplankton distribution in the Strait of Magellan. Bollettino di Oceanologia Teorica ed Applicata, 9, 145-150.

Valdenegro, A., \& Silva, N. (2003). Caracterización física y química de la zona de canales y fiordos australes de Chile entre el estrecho de Magallanes y el cabo de Hornos (CIMAR-3 fiordos). Revista Ciencia y Tecnología del Mar, 26 (2), 19-60.

Valle-Levinson, A., Blanco, J., \& Frangópulos, M. (2006). Hydrography and frontogenesis in a glacial fjord off the Strait of Magellan. Ocean Dynamics, 56, 217-227.

Vásquez, O., Pineda, S., Quiroga, E., Jara, E., \& Montiel, A. (2012). Relación entre clorofila-a y las variables oceanográficas en el área periglaciar del seno Gallegos (Cordillera Darwin, Chile): bajo condiciones invernales. Anales del Instituto de la Patagonia, 40, 139-151.

Venegas, C., Gibbons, J., Aguayo-Lobo, A., Sielfeld, W., Acevedo, J., Amado, N., Capella, J., Guzmán, G., \& Valenzuela, C. (2002). Distribución y abundancia de lobos marinos (Pinnipedia: Otariidae) en la Región de Magallanes, Chile. Anales del Instituto de la Patagonia, 30, 67-82.

Zingone, A., Sarno, D., Siano, R., \& Marino, D. (2011). The importance and distinctiveness of small-sized phytoplankton in the Magellan Straits. Polar Biology, 34 (9), 1269-1284. 